\title{
Assessment of the stresses produced on the bone implant/tissue interface to the different insertion angulations of the implant - a three-dimensional analysis by the finite elements method
}

\author{
Joelson-Rodrigues Brum 1, Fabiano-Rito Macedo ', Millena-Barroso Oliveira 2, Luiz-Renato Paranhos ${ }^{3}$, \\ Rui-Barbosa Brito-Júnior ${ }^{4}$, Juliana-Cama Ramacciato ${ }^{4}$ \\ ${ }^{1}$ DDs, MSc, PhD student, Faculdade São Leopoldo Mandic, Campinas, SP, Brazil \\ ${ }^{2}$ DDs, Post-Graduation Program in Dentistry, Federal University of Uberlândia (UFU), Uberlândia, MG, Brazil \\ ${ }^{3}$ DDs, MSc, PhD, Department of Community and Preventive Dentistry, Federal University of Uberlândia (UFU), Uberlândia, MG, \\ Brazil \\ ${ }^{4}$ DDs, MSc, PhD, Faculdade São Leopoldo Mandic, Campinas, SP, Brazil
}

Correspondence:

Department of Preventive and Community Dentistry

School of Dentistry, Federal University of Uberlândia

Uberlândia, Brazil. Pará Avenue, 1720, 2 G Block

Umuarama-38400902 Uberlândia/MG - Brazil

paranhos.lrp@gmail.com

Received: 20/05/2020

Accepted: 02/07/2020

Brum JR, Macedo FR, Oliveira MB, Paranhos LR, Brito-Júnior RB, Ramacciato JC. Assessment of the stresses produced on the bone implant/ tissue interface to the different insertion angulations of the implant - a three-dimensional analysis by the finite elements method. J Clin Exp Dent. 2020;12(10):e931-7.

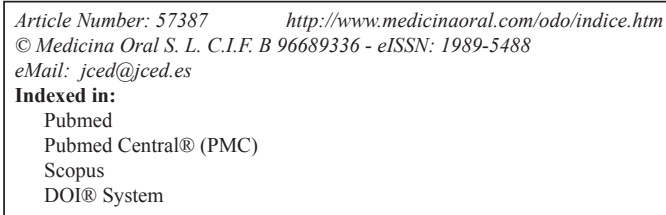

\begin{abstract}
Background: The present study aimed to assess the stresses produced on the surface of the bone tissue around dental implants with three different insertion angulations subjected to axial and oblique loading.

Material and Methods: The study was created according to the recommendations of the Checklist for Reporting In-vitro Studies (CRIS). The Straumann ${ }^{\mathrm{TM}}$ bone level RC $(4.1 \times 10 \mathrm{~mm})$ implant, Cone Morse connection $(\mathrm{CM})$, RC Straumann Variobase ${ }^{\mathrm{TM}}$ with abutment $(3.5 \mathrm{~mm})$ was placed in the region of element 16 , with the platform positioned at the height of the bone crest. Three assessment models were produced: model M1 or control - implant perpendicular to the bone crest; model M2 - implant angulated at $17^{\circ}$ relative to the bone crest; and model M3 - implant angulated at $30^{\circ}$ relative to the bone crest. The masticatory loads were simulated with $100 \mathrm{~N}$ of intensity and two loading patterns (axial and oblique) were applied to each model. Then, the models were exported to the finite elements simulation software Ansys Workbench V19.2 (Ansys Inc., Canonsburg, PA, USA). To assess the finite elements, qualitative and quantitative analyses were performed.

Results: It was observed that, under axial loading, qualitatively, the peaks occurred in the cavosurface region, palatal aspect in M1 and M2, and buccal aspect in M3. Quantitatively, the greatest angulation resulted in a low stress peak. Under oblique loading, qualitatively, the peaks occurred in the cavosurface region, buccal aspect in the three groups. Quantitatively, the greatest angulation of the implant resulted in an increase in stress peaks on the buccal aspect. Conclusions: Under axial loading, the three insertion angulations of the implant - M1, M2, and M3 - were clinically viable. When subjected to oblique loading, the $30^{\circ}$ angulation (M3) suggested a significant risk of bone loss and it was contraindicated.
\end{abstract}

Key words: Finite element analysis, dental implants, load support. 


\section{Introduction}

Implantology has been focusing studies in the search for technological and minimally invasive advancements both in the surgical phase (1-3) and the prosthetic and component selection phase (4). The analyses of the mechanical performance of implant-supported prostheses involve studies aiming not only to assess the resistance of the implant-prosthetic crown set (5) but also to understand the bone behavior $(5,6)$ to the potential variations of length, angulation, and implant positioning (7). Thus, investigations on design modifications, surface treatments, and screw spacing are performed daily (810) to prevent bone loss, consequently increasing the rate of success with dental implant treatments (8).

Ideally, implants for dental prostheses should be positioned parallel to each other but certain clinical circumstances require angulated implants (11), so understanding the bone response to different load applications becomes essential. Thus, the present study aimed to perform a three-dimensional analysis of the stresses produced on the surface of bone tissue around implants when subjected to axial and oblique loading. The null hypothesis tested in this study was that different insertion angulations of the implant would not affect the stress produced on the adjacent bone tissue.

\section{Material and Methods}

The local ethics committee approved the study (protocol no. 2020-0097). The entire study was created according to the recommendations of the Checklist for Reporting In-vitro Studies (CRIS) (12).
-Obtaining the geometric models

The three-dimensional model of the dentate and edentulous maxilla came from a pre-existing model available in the literature for the use of the scientific community (13). The models were downloaded in the parametric format using the SolidWorks $2011^{\mathrm{TM}}$ software. The geometric changes required in the model were performed in the CAD Solidworks 2017 software (Dassault Systemes, Solidworks Corps, USA).

To obtain the geometric models of the implant and the components used in the study, they were subjected to reverse engineering with a digital caliper (Mod. 500196-30B, Mitutoyo Sul Americana Ltda., Suzano, Brazil), digital microscope (MV500UM-PL, Cosview Technologies Co. Ltd, Bantian, China) with a magnification of 5x 200x, and a measuring software (Miviewcap 6.0, Cosview Technologies Co. Ltd, Bantian, China) to measure the geometry of the components and allow molding in the Solidworks software.

-Sample preparations

The Straumann ${ }^{\mathrm{TM}}$ bone level RC (4.1 x $10 \mathrm{~mm}$, Institut Straumann AG, Basel, Switzerland) implant, Cone Morse connection (CM), with RC Variobase ${ }^{\mathrm{TM}}$ abutment (3.5 mm, Institut Straumann AG, Basel, Switzerland) was placed in the region of element 16 with the platform positioned at the height of the bone crest, and it was considered osseointegrated. The position of the implant presented a variable angulation according to the model (Fig. 1): M1 or control - with the implant perpendicular to the bone crest; $\mathrm{M} 2$ - with the implant angulated at $17^{\circ}$
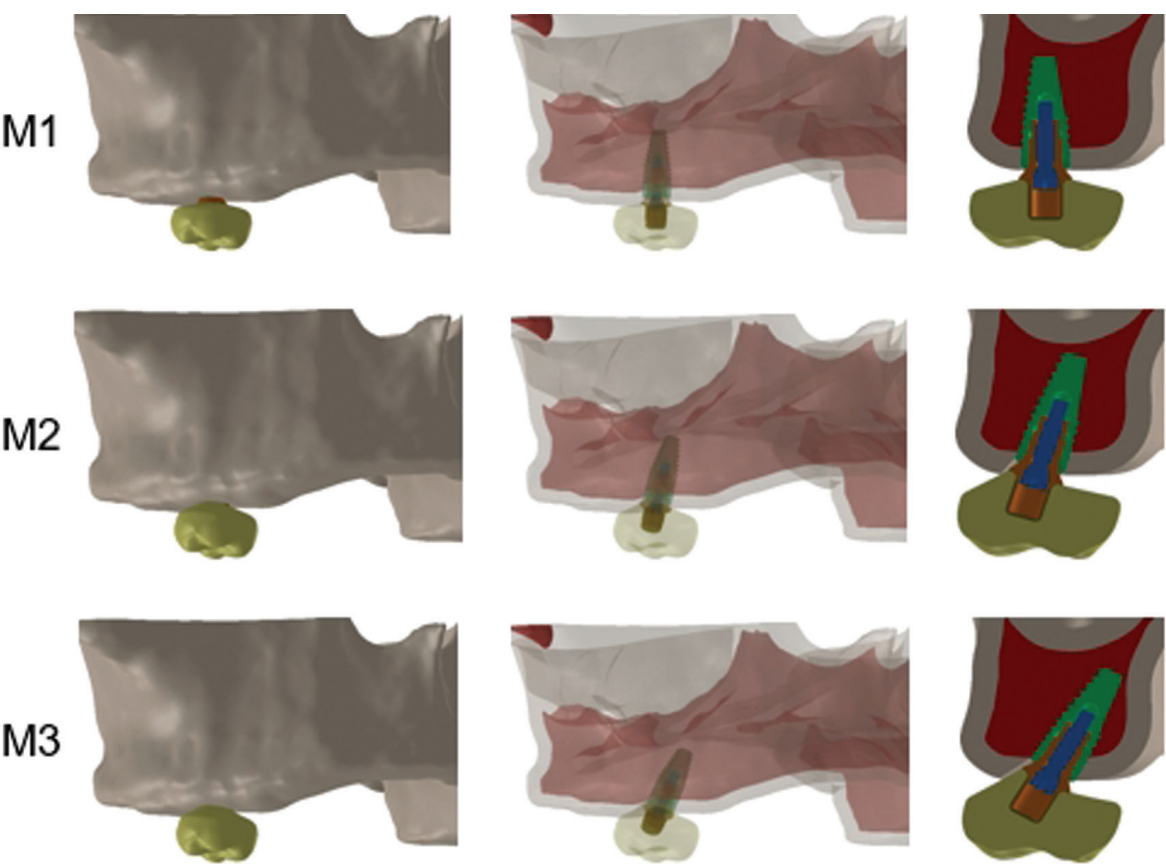

Fig. 1: Models analyzed with different angulations relative to the bone crest. M1 (perpendicular); M2 $\left(17^{\circ}\right)$; $\mathrm{M} 3\left(30^{\circ}\right)$. 
relative to the bone crest and apical portion facing the palate; and $\mathrm{M} 3$ - with the implant angulated at $30^{\circ}$ relative to the bone crest and apical portion facing the palate. The crown (lithium disilicate glass-ceramic, thickness of $1.5 \mathrm{~mm}$, IPS e.max press, Ivoclar, Vivadent, Schaan, Liechtenstein) was cemented with a layer of $62 \mu \mathrm{m}$ of resin cement between the restorative material and the prosthetic intermediate.

-Determination of contact points

The enamel structure that simulated the occlusal third of antagonist teeth received three circular contact points with $1 \mathrm{~mm}$ of diameter each, for axial loading application. One point was positioned in the buccal cusp and two other points in the palatal cusp, buccal and palatal sides. In turn, for applying the oblique loading, the points were positioned in the buccal sides of the lingual cusps. A bolus was added with the Young modulus similar to the almond, 21.57 (4.00) MPa (14) and a thickness of $2 \mathrm{~mm}$, between the ceramic crown and the antagonist structure of the enamel, as a way to represent a condition as naturally as possible.

-Load application

The axial loading was applied with a parallel vector along the axis of the element, over the upper portion of the structure that simulated the antagonist teeth. In the occlusal contact, the antagonist structure was set with frictionless supports on the sides to allow a uniquely gingival occlusal movement. The contacts between the antagonist structure and the crowns were set as "frictionless", allowing sliding and gap formation. This enabled both the intrusion and a buccolingual movement of the implants, similar to real-life conditions.

The oblique loading was simulated with a vector in the palatal-buccal direction, forming a $30^{\circ}$ angle with the occlusal plane. The antagonist structure was used to standardize the loading area.

Rigid supports were added in the areas in which the maxilla would connect to the rest of the skull. The simulations were non-linear relative to the contact.

-Finite element analysis

All models were exported to the finite elements simulation software Ansys Workbench V19.2 (Ansys Inc., Canonsburg, PA, USA) using an import accessory of the software itself.

To represent the mechanical behavior of each component as reliably as possible, the different elements of the models were set based on the elasticity modulus and Poisson's coefficient. All the material was considered linear, isotropic, and homogeneous. The implant (Roxo$\operatorname{lid}^{\mathrm{TM}}$ ) required the mean between the elasticity modulus of a titanium alloy with $10 \%$ zirconia and $90 \%$ titanium and another with $20 \%$ zirconia and $80 \%$ titanium (15).

Non-linear frictional contacts with a friction coefficient of $0.2 \mu$ (16) were used to simulate the contact between titanium surfaces. All the others were simulated with contacts that did not allow sliding or gap formation, except for the contact between the antagonist axial structure and the crown.

The masticatory loads were simulated with $100 \mathrm{~N}$ of intensity in the axial and oblique loading patterns.

The meshes of finite elements were created with a mesh refinement process until the variation reached $5 \%$ or less, indicating that the distortion by the mesh intensity would not affect significantly the results. The mesh was produced with quadratic tetrahedral elements of 10 knots (solid 187), which allowed copying the irregular geometry of the models analyzed. The number of knots/elements ranged from 1933932/1240795 to 1935240/1240714. All models were then resolved (Windows 1064 bits, Intel I7 6800k processor, 112 Gb RAM) and the graphic and numerical plots of the data were assessed and compared.

To assess the finite elements, qualitative and quantitative analyses were performed.

-Structural quantification of the risk of peri-implant bone damage

The Mohr Coulomb criterion was used to quantify at a structural level the risk of peri-implant bone damage. The Mohr Coulomb theory defines that material will fracture when the combination of the main stresses are equal or exceed the resistance limits. The impact of tensile stresses and its relationship with tensile strength were analyzed, as well as the compressive stresses and their relationship with compressive strength. To facilitate the comparative analyses, an adaptation was performed and defined by the formula: (Fig. 2).

$$
\frac{\sigma_{1}}{\sigma_{\text {limit yield to traction }}}+\frac{\sigma_{3}}{\sigma_{\text {limit yield to compression }}}=\sigma_{\mathrm{R}}
$$

Fig. 2: Formula.

where $\sigma_{\mathrm{R}}$ is the result, $\sigma_{1}$ is the main maximum stress, $\sigma_{3}$ is the main minimum stress, and $\sigma_{\text {limit }}$ represents the maximum yield stress to compression and traction. As a reference for the calculation, the limit yield stress to traction was $82.8 \mathrm{MPa}$ and the limit yield stress to compression was $133.6 \mathrm{MPa}$ (17). Such values were based on the trabecular bone, considering the reference values were not found within the same study for the indexes of cortical bone. As a convenience, model M1 was defined as control.

\section{Results}

Figure 3 shows a sectioned view of the peri-implant cortical insertions of models M1, M2, and M3.

When analyzed qualitatively, the results of the peri-implant bone under axial loading (Fig. 4) showed that the peaks occurred in the cavosurface region, on the palatal side of models M1 and M2 and the buccal side of model M3. Quantitatively, it was observed that the greatest angulation resulted in low stress peaks and consequently risk of bone loss. 

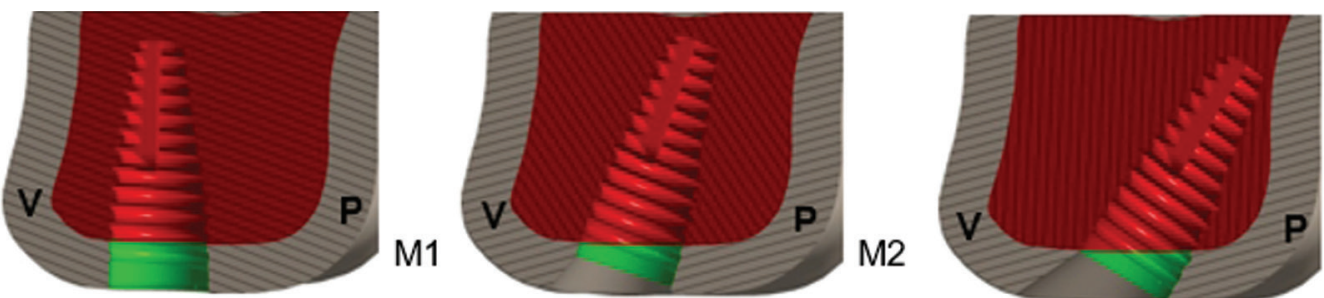

M3

Fig. 3: Peri-implant cortical bone insertion in the different models, highlighted in green. M1: perpendicular to the bone crest; M2: inclination of $17^{\circ}$; and M3: inclination of $30^{\circ}$.

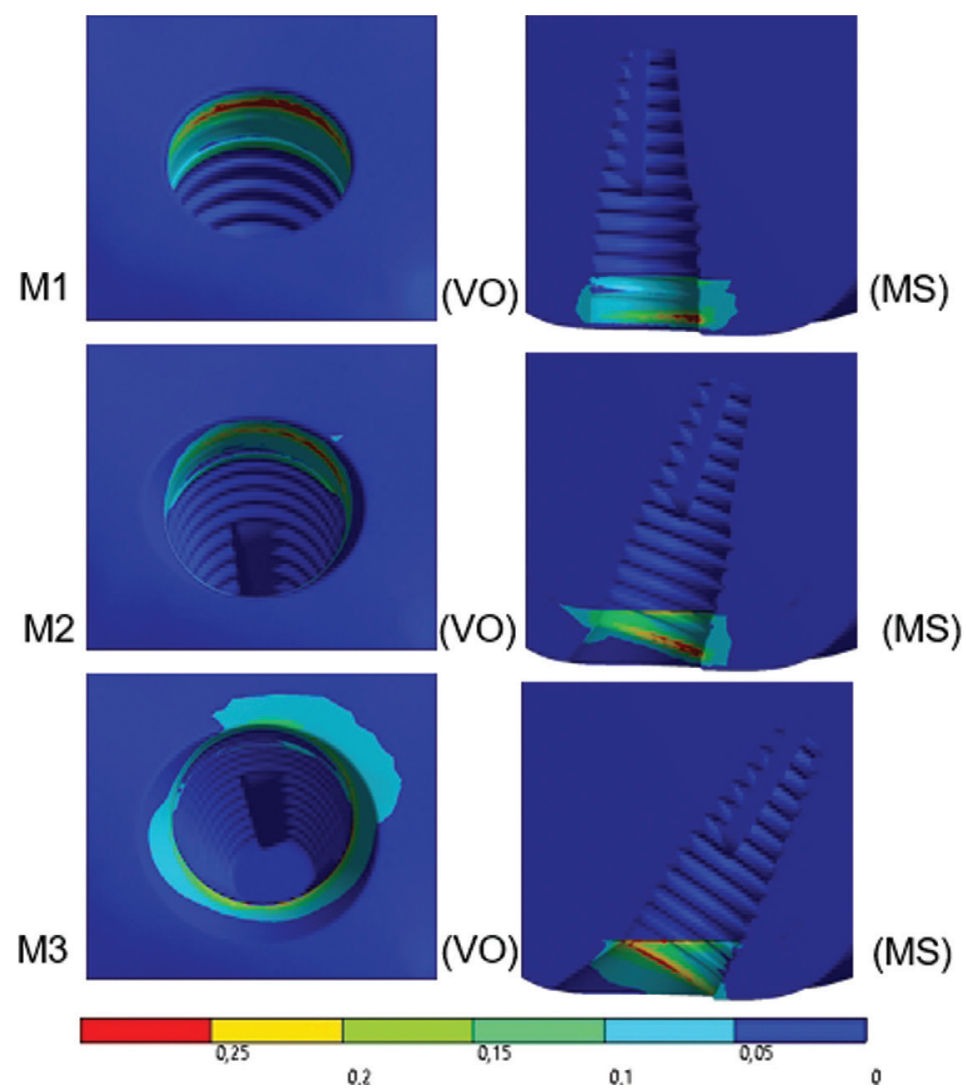

Fig. 4: External sectioned view of the results in the peri-implant bone under axial loading. A linear color scale was used, where blue indicates low values and red indicates high values of stress on the peri-implant bone. Models: M1, M2, and M3. VO view: buccal occlusal; MS: sectioned mesial. Considering the view is sectioned, the mesial view shows the distal portion.

When analyzing the results of the peri-implant bone under oblique loading qualitatively (Fig. 5), the peaks occurred in the buccal cavosurface region in all models. Quantitatively, there was an inversion of the trends found for the axial loading, with the greatest angulation of the implant increasing stress peaks.

The peaks of the results in the peri-implant bone, according to the Mohr Coulomb Criterion and its percentage, to the axial loading were: M1 - $0.362(100 \%)$; M2 - 0.351 (97\%); M3 - 0.332 (91\%). For the oblique loading, the results were: M1 $-0.741(100 \%)$; M2 0.763 (103\%); M3 - 1.208 (163\%).

\section{Discussion}

Three different insertion angulations of dental implants were simulated to assess the stress exerted on the adjacent bone when axial and oblique forces were applied. The hypothesis that different insertion angulations of the implant would not affect the stress produced on the adjacent bone tissue was rejected.

In the peri-implant bone under axial loading, qualitatively, the peaks occurred in the cavosurface region due to the closest proximity to the point of origin of the load in the cortical bone, affecting the palatal side in M1 $\left(0^{\circ}\right)$ and M2 $\left(17^{\circ}\right)$. In model M3 $\left(30^{\circ}\right)$, the positioning 


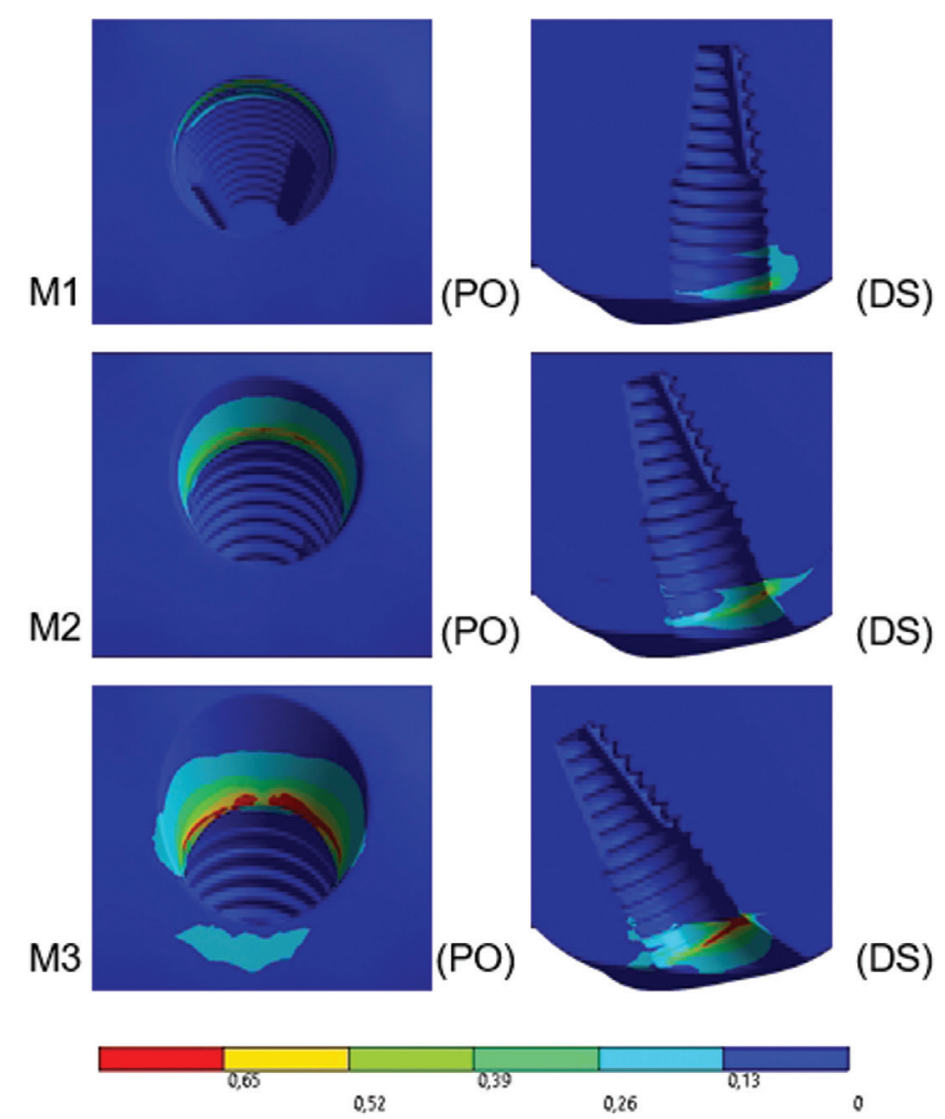

Fig. 5: External sectioned view of the results in the peri-implant bone under oblique loading. A linear color scale was used, where blue indicates low values and red indicates high values of stress on the peri-implant bone. Models: M1, M2, and M3. PO view: palatal occlusal; DS: sectioned distal. Considering the view is sectioned, the distal view shows the mesial portion.

of the peak was buccal and it may be explained by the thinning of the cortical bone in the buccal aspect due to the degree of angulation. Winter et al. (18) observed that the stability of implants in the models studied was positively correlated to the length and thickness of the cortical bone, validating the results found. Thus, according to Kurniawan et al. (19), for minimum stress and tension in a certain region, a peri-implant bone that is denser and osseointegrated is desired. As for the oblique loading, when analyzing the results of the peri-implant bone qualitatively, the peaks occurred in the buccal cavosurface region in all models, especially because the horizontal component of the oblique force is buccally directed, without divergences in the region affected among the models.

Quantitatively, the axial loading showed that the greatest angulation $\left(30^{\circ}\right)$ resulted in low stress peaks and the risk of bone loss. In turn, Amid et al. (20) observed that the application of an axial loading at $100 \mathrm{~N}$ and $30^{\circ}$ angle caused more stress when compared to the axial application of $300 \mathrm{~N}$ along the axis of implants, presenting different results from those found in the present study. For the oblique loading, quantitatively, there was an inversion of the trends found for the axial loading, in which the greatest angulation of the implant elevated the stress peaks. The data corroborate Amid et al. (20), who obtained maximum stress on the peri-implant bone when applying oblique loading at a $30^{\circ}$ angle.

Oblique loading and implant inclination were also harmful in the study by Almeida et al. (21), which showed that when increasing implant angulation, the area of stress concentration also increased on the adjacent bone, especially under the application of oblique loading. In the same study, the distribution of compressive and tensile stresses presented values of lower intensity for the axial loading.

In the structural quantification of the risk of peri-implant bone damage, by the Mohr Coulomb method, the difference of peaks among the models was a maximum of $9 \%$ for the axial loading. In the oblique loading, the difference was $3 \%$ between models M1 (100\%) and M2 $(103 \%)$, suggesting a similar clinical performance acceptable between angulations. However, in model M3, the difference was $63 \%$ under oblique loading, showing 
a higher risk of bone loss with the increase of insertion angulation. In line with the findings, Hong et al. (7) observed in their study that as the implants were inclined, not only the levels of stress on the bone tissue increased but also the efficiency of stress distributions on the adjacent bone decreased. The authors also showed that the lowest stress and the best implant stability on mandibular overdentures were obtained when the implants were inserted in the regions of lateral incisors with shorter implants positioned parallel to the long axis of the teeth (7). Hence, M3 was contraindicated.

The implant model M1 presented the best results for stress on the peri-implant bone tissue when compared to models M2 and M3. This is mainly because the type and position of the implant affect directly the levels of stress produced, thus the implants placed along the loading axis show a better stress distribution (22). Watanabe et al. (23) verified that, regardless of the point and direction of the load application, the compressive stresses were relatively higher when the implant was inclined. Thus, when the load was applied with a direction of 45 degrees, the compressive stress on the cortical bone was adjacent to the implant inclination, as the elastic stress was restricted only to the opposite side.

For the clinical decision, the findings of the present study were rather significant because they showed that the relationship between insertion angulation of the implant and the load applied, in this case axial or oblique, affected directly the stress on the surrounding bone tissue. Studies show that the maximum equivalent stress increases linearly to the load angle increase, that is, for each increase of 30 degrees in the load angle, the maximum equivalent stress on the cortical bone increases in average 3 to 4 times in comparison to the axial loading applied (24).

As a limitation, the model of finite element analysis of this study could not simulate all the characteristics of the living tissue, although the simulation with bolus between the occlusal third of the ceramic crown and the antagonist structure of the enamel was able to affect which regions the peaks of axial and oblique loading applied would occur.

The finite element analysis allowed a satisfactory in vitro model simulation through the numerical/computer reproduction of the structures and materials involved in this study. Therefore, it is evident the significance of in vitro studies as a key point to a coherent clinical indication and predictability. This study showed that different insertion angulations, according to models M1, M2, and M3, caused different levels of stress on the underlying bone tissue when the models were subjected to axial and oblique loading. However, further clinical research should be performed to approximate the results found to the behavior of living tissues.

\section{Conclusions}

The insertion angulations of the implant studied were clinically viable when applying axial loading. When subjected to oblique loading, the $30^{\circ}$ angulation suggested a significant risk of bone loss and its clinical application should not be recommended.

\section{References}

1. Pisoni L, Ordesi P, Siervo P, Bianchi AE, Persia M, Siervo S. Flapless versus traditional dental implant surgery: long-term evaluation of crestal bone resorption. J Oral Maxillofac Surg. 2016;74:1354-9.

2. Slete FB, Olin P, Prasad H. Histomorphometric comparison of 3 osteotomy techniques. Implant Dent. 2018;27:424-8.

3. Divakar TK, Gidean Arularasan S, Baskaran M, Packiaraj I, Dhineksh Kumar N. Clinical evaluation of placement of implant by flapless technique over conventional flap technique. J Maxillofac Oral Surg. 2020;19:74-84

4. Meriç G, Erkmen E, Kurt A, Eser A, özden AU. Biomechanical effects of two different collar implant structures on stress distribution under cantilever fixed partial dentures. Acta Odontol Scand. 2011;69:374-384.

5. Abu-Hammad O, Khraisat A, Dar-Odeh N, Jagger DC, Hammerle $\mathrm{CH}$. The staggered installation of dental implants and its effect on bone stresses. Clin Implant Dent Relat Res. 2007;9:121-7.

6. Behnaz E, Ramin M, Abbasi S, Pouya MA, Mahmood F. The effect of implant angulation and splinting on stress distribution in implant body and supporting bone: a finite element analysis. Eur J Dent. 2015;9:311-8.

7. Hong HR, Pae A, Kim Y, Paek J, Kim HS, Kwon KR. Effect of implant position, angulation, and attachment height on peri-implant bone stress associated with mandibular two-implant overdentures: a finite element analysis. Int J Oral Maxillofac Implants. 2012;27:e6976.

8. Karoussis IK, Brägger U, Salvi GE, Bürgin W, Lang NP. Effect of implant design on survival and success rates of titanium oral implants: a 10-year prospective cohort study of the ITI dental implant system. Clin Oral Implants Res. 2004;15:8-17.

9. Eroglu CN, Ertugrul AS, Eskitascioglu M, Eskitascioglu G. Changes in the surface of bone and acid-etched and sandblasted implants following implantation and removal. Eur J Dent. 2016;10:77-81.

10. Khorsand A, Rasouli-Ghahroudi AA, Naddafpour N, Shayesteh YS, Khojasteh A. Effect of microthread design on marginal bone level around dental implants placed in fresh extraction sockets. Implant Dent. 2016;25:90-6.

11. Lan TH, Pan CY, Lee HE, Huang HL, Wang CH. Bone stress analysis of various angulations of mesiodistal implants with splinted crowns in the posterior mandible: a three-dimensional finite element study. Int J Oral Maxillofac Implants. 2010;25:763-770.

12. Krithikadatta J, Gopikrishna V, Datta M. CRIS guidelines (checklist for reporting in-vitro studies): a concept note on the need for standardized guidelines for improving quality and transparency in reporting in-vitro studies in experimental dental research. J Conserv Dent. 2014;17:301-4.

13. Vasco MAA, Souza JTA, Las Casas EB, de Castro e Silva ALR, Hecke M. A method for constructing teeth and maxillary bone parametric model from clinical CT scans. Comp Method Biomech Biomed Enginee: Imag Visual. 2015;3:117-122.

14. Agrawal KR, Lucas PW, Prinz JF, Bruce IC. Mechanical properties of foods responsible for resisting food breakdown in the human mouth. Arch Oral Biol. 1997;42:1-9.

15. Ho WF, Chen WK, Wu SC, Hsu HC. Structure, mechanical properties, and grindability of dental Ti-Zr alloys. J Mater Sci Mater Med. 2008; 19:3179-3186.

16. Jörn D, Kohorst P, Besdo S, Rücker M, Stiesch M, Borchers L. Influence of lubricant on screw preload and stresses in a finite element model for a dental implant. J Prosthet Dent. 2014;112:340-8.

17. Bayraktar HH, Morgan EF, Niebur GL, Morris GE, Wong EK, 
Keaveny TM. Comparison of the elastic and yield properties of human femoral trabecular and cortical bone tissue. J Biomech. 2004;37:27-35. 18. Winter W, Möhrle S, Holst S, Karl M. Parameters of implant stability measurements based on resonance frequency and damping capacity: a comparative finite element analysis. Int J Oral Maxillofac Implants. 2010;25:532-9.

19. Kurniawan D, Nor FM, Lee HY, Lim JY. Finite element analysis of bone-implant biomechanics: refinement through featuring various osseointegration conditions. Int J Oral Maxillofac Surg. 2012;41:1090-6. 20. Amid R, Rasoolzadeh RA, Motlagh AM, Dehnavi F, Kadkhodazadeh M. Stress and strain distribution patterns in bone around splinted standard and short implants placed at the crestal level and subcrestally using Three- Dimensional finite element analysis. J Long Term Eff Med Implants. 2017;27:1-11.

21. de Faria Almeida DA, Pellizzer EP, Verri FR, Santiago JF Jr, de Carvalho PS. Influence of tapered and external hexagon connections on bone stresses around tilted dental implants: three-dimensional finite element method with statistical analysis. J Periodontol. 2014;85:261269.

22. Lin CL, Chang SH, Chang WJ, Kuo YC. Factorial analysis of variables influencing mechanical characteristics of a single tooth implant placed in the maxilla using finite element analysis and the statistics-based Taguchi method. Eur J Oral Sci. 2007;115:408-416.

23. Watanabe F, Hata Y, Komatsu S, Ramos TC, Fukuda H. Finite element analysis of the influence of implant inclination, loading position, and load direction on stress distribution. Odontology. 2003;91:31-6.

24. Hsu ML, Chen FC, Kao HC, Cheng CK. Influence of off-axis loading of an anterior maxillary implant: a 3-dimensional finite element analysis. Int J Oral Maxillofac Implants. 2007;22:301-9.

\section{Source of Funding}

This study was also financed in part by CAPES (Coordenação de Aperfeiçoamento de Pessoal de Nível Superior - Brazil) - Finance Code 001. We are also thankful for the support of CNPq (Council for Scientific and Technological Development - Brazil) - Finance Code 307808/2018-1.

\section{Ethics}

Not applicable.

\section{Conflict of interest}

None. 